\title{
The Areas Under Curves (AUC) used in diabetes research: Update view
}

\author{
Cheng $\mathrm{KC}^{1}$, Li $\mathrm{Y}^{1,2}$ and Cheng JT ${ }^{2 *}$ \\ ${ }^{1}$ Department of Psychosomatic Internal Medicine, Kagoshima University Graduate School of Medical and Dental Sciences, Kagoshima, Japan \\ ${ }^{2}$ Department of Medical Research, Chi-Mei Medical Center, Yong Kang, Tainan City, Taiwan
}

\begin{abstract}
The Area Under the Curve (AUC) is derived from the Oral Glucose Tolerance Test (OGTT) which is widely used to diagnose the Impaired Glucose Tolerance (IGT) in the clinic. Due to the variations in baseline blood glucose, the incremental Area Under Curve (iAUC) is developed. But, subtracting the baseline value of fasting plasma glucose to have the iAUC has been criticized. Thus, we are interested to understand the advantage and limitations of iAUC in clinical practice and basic research. It seems used in a way not better than original AUC (tAUC) both from the mathematical view and clinical reports. Moreover, iAUC was widely applied in the calculation of the Glycemic Index (GI) of food. But, it limited in the healthy volunteers received food immediately. Otherwise, iAUC is not suitable to apply in basic research because the animals used are generally maintained in constant environment to receive standard chow and water. Therefore, variations in fasting plasma glucose are markedly less than the human subjects. Additionally, iAUC is contraindicated to apply in the animals received chronic treatment that is widely used in basic research. Overall, iAUC is suitable to apply in clinic under careful manner. Thus, we recommend applying the AUC without modification (tAUC) during OGTT both in bench and bedside.
\end{abstract}

\section{Backgrounds}

In 1995, one technical article titled "The use of areas under curves in diabetes research" has been published [1]. It criticized the abuse of incremental area under curve (iAUC). Now, we are interested to revisit this title using the update view.

The area under the curve (AUC) is derived from the oral glucose tolerance test (OGTT) which is widely used to diagnose the impaired glucose tolerance (IGT) in the clinic. It is going to estimate the total rise in blood glucose during OGTT, calculating from the trapezium rule, trapezoidal method, or composite trapezoidal method as described previously [1]. But, AUC is usually calculated using the trapezoidal rule now. The application of iAUC is developed due to the variations in fasting plasma glucose between individuals. But, the iAUC from subtracting the baseline value of fasting plasma glucose has been challenged as problematic [1] due to the formation of negative value(s). Therefore, the positive incremental AUC (pAUC) has further been suggested and only the values above the baseline value were considered to apply in the studies $[2,3]$. On the other word, the total area under the curve (tAUC), incremental area under the curve (iAUC), and positive incremental area under the curve (pAUC) were applied in clinical practice. However, the cut-off baseline values (iAUC) are still concerned, either in the mathematical view [1] or from the clinical report [4]. Recently, it has been indicated that tAUC expressed the best correlation with the 2-hour glucose level of OGTT and the total glucose response was represented by the tAUC better than iAUC or pAUC in a clinical report [5]. The advantage of iAUC seems limited in the clinical practice.

\section{Application of iAUC in clinical practice}

The iAUC value is also applied to calculate the Glycemic Index (GI) of food in nutrition [6]. The invited volunteer(s) were the healthy subjects to participate in the study. Application of iAUC is going to minimize the difference between individuals. But the variations in fasting plasma glucose between healthy subjects are not such critical as diabetic patients. Moreover, the GI value is calculated as a percentage of the reference food (glucose). Therefore, the application of iAUC shall be careful, particularly it is suitable to distinguish the change(s) in cases after an acute administration, similar to the postprandial glycemic responses.

In clinics, the variations in fasting plasma glucose between diabetic patients are associated with the pathogenic condition. It has been established that diabetic disorders are progressive [7]. Thus, fasting plasma glucose is varied mainly with the diabetic progress. The difference depends on both the between- and within-person variability. Therefore, application of iAUC in diabetic patients must keep in mind to conduct the associated factors carefully.

\section{Limitations of iAUC in basic research}

In basic research, the used animals were maintained in wellcontrol condition to receive standard chow and water through inbreed generation. Thus, iAUC is not widely used in basic research. Particularly, iAUC is contraindicated to apply in the animals received chronic treatment that is widely used in the assay of bioactivities.

${ }^{\star}$ Correspondence to: Juei-Tang Cheng, Department of Medical Research, ChiMei Medical Center, Yong Kang, Tainan City, Taiwan, Tel: + 886-6-251-7864, E-mail: jtcheng5503@gmail.com

Key words: areas under curves (AUC), incremental AUC (iAUC), oral glucose tolerance test (OGTT), basic research, clinical practice

Received: September 10, 2018; Accepted: September 20, 2018; Published: September 25, 2018 
According to our previous report [8], no paper subtracted the values of fasting plasma glucose or basal glucose level to calculate the AUC during OGTT in the recent 10 years (2007-2017). The chronic method is performed daily with repeated treatment and it may include many factors more complicated than acute treatment. Herbal extract, the new agent, or nutrient is usually applied to treat with diabetic animals once or several times daily at the desired dose. The test substance is repeatedly treated for one week or more to mimic the real applications. The treated diabetic animals were then used to receive OGTT. Mostly, the fasting plasma glucose is significantly modified in these animals indicating the influence of test substance [9]. The influenced fasting plasma glucose has been introduced to associate with the changes in hepatic glucose homeostasis [10]. Therefore, change in fasting plasma glucose was mainly derived from the cumulated effects of the test substance after a repeated treatment in animals. The derived fasting plasma glucose level, regardless of the degree of modification, shall be included in the calculation of AUC. Depending on this truth, iAUC is not available in animals received chronic treatment of test substance. Additionally, iAUC is also not suitable to minimize the variations in fasting plasma glucose between genetic mice and the wild-type littermates, as described previously [11], similar to the difference between diabetic model and normal group.

Mathematically, iAUC is similar to indicate as $\triangle \mathrm{AUC}$. But, $\triangle \mathrm{AUC}$ is widely applied in pharamcokinetics for another way. Therefore, iAUC is more popular than $\triangle \mathrm{AUC}$ to apply in diabetic research.

\section{Conclusion}

Taken together, it seems better to apply the no modified AUC (tAUC) in basic research. Application of iAUC seems limited in the cases receiving acute treatment only, both in bench and bedside. Although iAUC may be helpful to minimize the variations in fasting plasma glucose or baseline blood glucose, it shall be applied in a careful way because of the difference in some cases raised from the pathologic condition. Nevertheless, iAUC cannot be used in animals received chronic treatment of test substance.

\section{Acknowledgement}

We thank Miss Y.L. Yen for the kindly help in the collection of references.

\section{References}

1. Allison DB, Paultre F, Maggio C, Mezzitis N, Pi-Sunyer FX (1995) The use of areas under curves in diabetes research. Diabetes Care 18: 245-250. [Crossref]

2. Wolever TM, Jenkins DJ, Jenkins AL, Josse RG (1991) The glycemic index: methodology and clinical implications. Am J Clin Nutr 54: 846-854. [Crossref]

3. Wolever TM, Jenkins DJ (1986) The use of the glycemic index in predicting the blood glucose response to mixed meals. Am J Clin Nutr 43: 167-172. [Crossref]

4. Sainaghi PP, Castello L, Limoncini AM, Bergamasco L, Bartoli E, et al. (2007) Poor specificity of fasting plasma glucose cut-off values in ruling out glucose intolerance: the complementary usefulness of OGTT. Exp Clin Endocrinol Diabetes 115: 112-117. [Crossref]

5. Khan A, Hornemann T (2017) Correlation of the plasma sphingoid base profile with results from oral glucose tolerance tests in gestational diabetes mellitus. EXCLI J 16: 497-509. [Crossref]

6. Jenkins DJ, Wolever TM, Taylor RH, Barker H, Fielden H, et al. (1981) Glycemic index of foods: a physiological basis for carbohydrate exchange. Am J Clin Nutr 34: 362-366. [Crossref]

7. Rahier J, Guiot Y, Goebbels RM, Sempoux C, Henquin JC (2008) Pancreatic betacell mass in European subjects with type 2 diabetes. Diabetes Obes Metab 4: 32-42. [Crossref]

8. Kuo SC, Li Y, Cheng JT (2018) Glucose tolerance test applied in screening of antidiabetic agent(s). Curre Res Diabetes \& Obes J 7: 555761

9. Ren B, Qin W, Wu F, Wang S, Pan C, et al. (2016) Apigenin and naringenin regulate glucose and lipid metabolism and ameliorate vascular dysfunction in type 2 diabetic rats. Eur J Pharmacol 773: 13-23. [Crossref]

10. Petersen MC, Vatner DF, Shulman GI (2017) Regulation of hepatic glucose metabolism in health and disease. Nat Rev Endocrinol 13: 572-587. [Crossref]

11. Cheng KC, Li Y, Cheng JT (2018) Merit of incremental area under the curve (iAUC) in nutrition is varied in pharmacological assay- A Review. Clin J Dia Care Control 1 : 180008 .

Copyright: (C2018 Cheng KC. This is an open-access article distributed under the terms of the Creative Commons Attribution License, which permits unrestricted use, distribution, and reproduction in any medium, provided the original author and source are credited. 